Commun. math. Phys. 1, 6-13 (1965)

\title{
Consistency of Relativistic Particle Theories
}

\author{
By \\ H. EKsTEIN * \\ University of Aix-Marseille, France
}

\begin{abstract}
While direct-interaction particle theories are generally thought to be incompatible with relativity in classical physics, such relativistic theories in quantum mechanics have recently attracted attention. The reasons for rejecting these theories in classical physics are based on the consideration of world lines, while relativistic quantum mechanics has no covariant position operator so that the classical refuting argument cannot be adapted.

This paper discusses the consistency of relativistic particle theories with a finite number of degrees of freedom, without recourse to the position operator. A particle is described by a sub-algebra of observables at one time. Homogeneous transformations, including "accelerations," must preserve the identity of particles, and therefore leave the sub-algebras invariant. It is shown that with this assumption only non-interacting particle theories are compatible with the principle of relativity, in quantum as well as classical mechanics.
\end{abstract}

\section{Introduction}

Relativistic quantum mechanical particle theories [1-4] - as contrasted to field theories - have recently attracted great attention, because they make phenomenological two- or three-particle problems amenable to a rigorously relativistic, mathematically consistent treatment. On the basis of classical considerations, it had long been believed that the relativistic interaction of two particles required a field, i.e. infinitely many variables. Hence, the new developments are somewhat surprising. Is it possible that quantum mechanics can succeed in a direct-interaction relativistic theory where classical physics fails, and if so, what are the virtues of quantum mechanics which make it possible?

Closer inspection shows that the success of quantum mechanical particle theories is bought for a price: the particle variables $q_{i}$ are the Wigner-Newton position operators which do not "transform covariantly" and therefore cannot be seriously considered as the quantum mechanical equivalent of the classical position variable $x_{\mu}(\mu=1$ to 3$)$. This circumstance raises a question of principle, both in classical and in quantum mechanics: is the instantaneous position of a particle a necessary and

* Permanent address: Argonne National Laboratory, Argonne, Illinois. 
a well-defined concept? Empirically, we can, of course, measure position of elementary particles only with great inaccuracy, and the extrapolation to a precise position may be a useless idealization.

In the literature, another observable, the "centroid of energy," has been discussed repeatedly [5]. This observable has the desired transformation properties and it is, in the low-energy limit, equal to the position times the mass. However, the three components do not commute, and it is therefore not usually considered as a proper relativistic generalization of the position. Indeed, if the three components of the position operator did not commute, it would not be possible to localize a particle, i.e. to create states in which the uncertainty of position is arbitrarily small. However, we might accept the impossibility of such a localization, if it leads to useful results.

Instead of investigating specific alternatives for position operators, we examine, more generally, the possibility of relativistic particle theories in which the single-particle observables are not necessarily given a physical interpretation. Of course, such theories can be only provisional, since the predictive power of a theory depends on physical interpretation of a complete set of observables. But, as Einstein remarked, it is possible that the full physical interpretation of a theory follows its formal development after years or even decades.

In this framework, the usual arguments against classical relativistic theories of interacting particles do not necessarily hold: the argument which uses the relativistic principle of finite signal velocity to exclude instantaneous interaction between distant particles is based on the idea that particles are points with well-defined positions *. Similarly, the argument given recently by CURRIE, JORDAN and SUDARSHAN [6], which does not use the principle of finite signal velocity, is also based on the physical interpretation of the operator $q_{i}$ (the canonical conjugate of the momentum) as position operator, and it loses its point if this physical interpretation is abandoned. We give a definition of the particle concept which, while in agreement with existing theoretical concepts, is more general. In intuitive language, our definition of particle requires only that it preserves its individuality under Lorentz or Galilei transformations. Our result is negative: even if the position concept is entirely abandoned, relativistic particle theories with interaction are not physically consistent, either in classical or quantum mechanics, or even in a larger class of general schemes which includes classical and quantum mechanies.

* By "theory" we mean prediction based on initial data, and not "closed systems" as defined by P. Havas: "Relativity and Causality" (Paper presented at the International Congress for Logic, Methodology and Philosophy of Science, Jerusalem, 1964.) 


\section{Assumptions and definitions}

We give a general framework for observables and symmetry (covariance) which is general enough to cover both classical and quantum mechanics. This has the advantage that we do not have to give separate arguments for the two cases. It also has the rather academic advantage that it covers a class of possibles theories which are more general than either classical or quantum mechanics.

An observable is a class of procedures with an apparatus, characterized by a space-time volume $V$ or a time $t$ (space-like hyperplane $\sigma$ ) within which the observation takes place. If two procedures, characterized by the same $V$ or $\sigma$, have the same expectation values with respect to all states (ensembles), they belong to the same equivalence class. (We are using the "Heisenberg concept" of observables, where states are timeindependent.)

An equivalence class of observables which have the same expectation values with respect to all states - and in general belong to different $V$ or $\sigma-$ is called a dynamical variable [7]. We assume that the observables form an algebra, without specifying the multiplication: it may be commutative as in classical physics or a Jordan product, as in quantum mechanics. We do not define a topology.

We consider, in particular, those observations which are made at one instant over all space, e.g. position, momentum, energy. By moving the apparatus from its initial position, a new observable is obtained. We shall consider the following motions of apparatus: translations, rotations and "accelerations," i.e. the apparatus is put into a state of uniform translation with respect to its original state. Finally, we consider "timetranslation" as a motion. This consists in changing the delay between some event characteristic for the production of the state and an instant characteristic of the measurement. In the space of all observables, a motion induces a permutation. We assume, in accordance with experience, that this permutation is an automorphism, i.e., it preserves algebraic relations, e.g.

$$
A \rightarrow A^{\prime}, \quad B \rightarrow B^{\prime}
$$

imply that

$$
\begin{gathered}
A+B \rightarrow A^{\prime}+B^{\prime}, \\
A^{2} \rightarrow A^{\prime 2} \text { etc } . .
\end{gathered}
$$

If a succession of two motions is defined as multiplication, the motions form a group, since every motion can be reversed.

Two distinct motions, e.g. the sequence $L_{1} L_{2}$ and the motion $L_{3}$ may have identical results as to the expectation values with respect to all states. Then, the two motions $L_{1} L_{2}$ and $L_{3}$ generate identical auto- 
morphisms of dynamical variables, and we will write

$$
L_{1} L_{2}=L_{3} \text {. }
$$

One can study the multiplication table of the group empirically. We are going to consider the Galilei and Poincaré groups. It is convenient to consider the realization of these groups by transformations of space-time. This realization associates to each motion $L$ a transformation which is intuitively obvious for all but "acceleration." To an acceleration, the Galilei group associates a transformation

$$
x^{\prime}=x+v t, t^{\prime}=t
$$

while the Poincaré group associates it to Lorentz transformations.

The set of observables $\{A(\sigma)\}$ associated to the hyperplane $\sigma$ has a specific structure: every dynamical variable has an associated element $A(\sigma)$, i.e. the set $\{A(\sigma)\}$ forms a closed algebra, and it is mapped onto the whole of the space of dynamical variables. This assumption is necessary in classical physics as well as quantum mechanics in order to guarantee (conventional) causality [7]. The preceding assumptions are common to almost all general frameworks for physical theory, and need no particular discussion. We consider now more specifically observables which characterize particles.

For simplicity, we restrict our discussion to a finite number of distinguishable particles, but the latter restriction is not essential.

A system of $N$ distinguishable particles is characterized by the fact that the algebra $\{A(\sigma)\}$ is generated by subalgebras $\left\{A_{i}(\sigma)\right\}(i=1 \ldots N)$ which have only the zero and unit elements in common. Since we have assumed the time-translation to induce an automorphism $A(\sigma) \rightarrow A(\sigma+t)$, the structure of $\{A(\sigma)\}$ is that of each $\{A(\sigma+t)\}$.

Physically, this assumption means first that the observables characterizing the different particles are independent of each other, i.e. that the specification of expectation values of the observables of particle 1 does not restrict the expectation values of the observables of particle 2 . For, if the algebras $\left\{A_{i}(\sigma)\right\}$ had a non-trivial intersection, one could express a member of $\left\{A_{j}(\sigma)\right\}$ as a function of the members of $\left\{A_{i}(\sigma)\right\}$ $(i \neq j)$.

Our assumption has the further physical implication that there are no observables other than particle observables necessary to predict the future. This specification excludes mediating fields, whose instantaneous properties would influence particle behavior in the future. The only field allowed by our specification is one which does not interact with the particles or (trivially) one which is defined as a given function of particle observables. For instance, a rigid self-field attached to each particle would still be in agreement with our assumption. 
Clearly, all known classical and quantum theories of particles satisfy the requirement.

The subalgebras $\left\{A_{i}(\sigma)\right\}$ have the further property that they are invariant under "space-translation" within the hyperplane $\sigma$. This, again, is obvious in classical and quantum mechanics. A space-translation changes e.g. $q_{i}$ into $q_{i}+a$, but never into a function of $q_{j}(j \neq i)$. If it were not true, the properties of a given particle, as seen by a translated observer, would not be fully determined by the properties of that particles, as seen by a static observer; they would depend on the observables of other particles.

Our next assumption is that the subalgebras $\left\{A_{i}(\sigma)\right\}$ are invariant under all elements $L$ of the homogeneous Galilei or Poincaré groups. Operationally, this statement means that there cannot be two particles which have all properties in common, as viewed from a fixed frame, but differ in their properties when viewed from a rotated or moving frame.

Classical relativistic particle theories traditionally appear in tensor or spinor form. We assert that the (usually unstated) physical reason for this formalism is that the above assumption is tacitly acknowledged to be the part of the definition of a particle. It implies that the instantaneous properties of a given particle, as viewed by a moving observer, can be calculated in a particularly simple way from the properties of that same particle as viewed by a static observer.

To appreciate the operational meaning of our assumption, consider an experiment designed to test it. Given two states, and given that the expectation values of all observables referring to particle $n^{0} 1$ are the same, we consider the expectation values of dynamical variables $L$ $A_{1}(\sigma)$ where $L$ is an acceleration, i.e. the results of observations made with the same instruments after they have been accelerated. Is it possible that these observables $L A_{1}(\sigma)$ are different in the two cases, i.e. can the state of particle 1, as viewed from a different frame at a given instant, depend on other particles? It seems that such an occurence would be considered as evidence for the fact that the system under observation is more complicated than a particle: e.g. a point particle surrounded by a "deformable" cloud or field. In this case, we could well understand that the properties of the system are not exhaustively described by instantaneous observations; since the accelerated frame sees different spacetime points as simultaneous it would not be surprising that the observables $L A_{i}(\sigma)$ are not fully determined by the set of $A_{i}(\sigma)$. Then, we would not describe the situation in terms of a finite number of particles, but in terms of a field, or, perhaps, clouds with an indefinite number of bare particles. On other words: the test for the particle nature of an object is that its identity is preserved under Lorentz transformations. 


\section{Conclusion and discussion}

We now have the following situation to consider: the automorphisms induced by space translations and by homogeneous transformations leave the subalgebras $\left\{A_{i}(\sigma)\right\}$ invariant. Can the remaining subgroup, i.e. the time translations, $\left\{T_{\tau}\right\}$ carry observables $A_{i}(\sigma)$ into dynamical variables associated to $B_{j}(\sigma)$ with $i \neq j$, i.e. can a time-translation mix the subalgebra $\left\{A_{i}(\sigma)\right\}$ ? If not, we have a trivial case: each particle behaves as though it were alone, and there is no interaction. In the case of the Galilei group the answer to our question is, of course, positive, as shown by nonrelativistic particle theories. For the Poincaré group, we have the following statement which is intuitively obvious, but proved in the Appendix.

If a function on the group is constant on the subgroups: space-translation and homogeneous subgroup, then it is constant on the whole group. Hence, the time translation leaves the subalgebras $\left\{A_{i}(\sigma)\right\}$ also invariant: the only relativistic particle theory is one of free (noninteracting) particles.

The argument of Currie, Jordan and Sudarshan [6] is a special case of the present conclusion. Their Eq. (3.18) is based on the interpretation of $Q^{n}$ as position of the $n$ 'th particle and it specifies the permutation of the elements of $\left\{A_{n}(\sigma)\right\}$ (including position and velocity) induced by an acceleration. As we have seen, the only feature essential for the conclusion is the existence of such a permutation, i.e. the invariance of algebra $\left\{A_{n}(\sigma)\right\}$ under Lorentz transformations.

Our conclusions are not in contradiction with F. Coester's view [4] on relativistic particle theories. Coester considers only the scattering operator as physically significant and attributes no observable meaning to the operators which tend asymptotically toward the in- and outoperators.

The negative conclusion does not destroy the value of relativistic particle theories as a provisional approach, it only delimits their claim to legitimacy. It may even be hoped that this clarification can improve the relativistic particle theories in a way which we discuss now.

The most striking pragmatic defect of reletivistic particle theories - at least for the case of two particles - is the lack of constraints on the interaction potential. Far from implying e.g. the correct form of the hydrogen spectrum, they allow for instance, a Coulomb interaction which leaves the spin invariant. One shudders at the thought of what would have happened to physics if DIRAC had known about the BakamjianThomas-Foldy type of relativistic particle theories. It seems imperative to find a restrictive principle to select the empirically correct interactions. It was originally the search for this additional restricting principle which 
motivated the present investigation. We found, disappointingly, that the rigorous application of the additional physical requirement: (the definition of particles) restricts the relativistic particle theories to the trivial case. It seems tempting to make a pragmatic use of this finding by requiring relativistic particle theories to satisfy approximately the requirements which we know, they cannot rigorously satisfy, thereby restricting the class of allowable interaction operators.

The author expresses his gratitude to D. KASTLER both for his hospitality and stimulating discussions.

\section{Appendix}

Given a representation of either of the two groups of motions (GALILEI and Poncaré), we consider the subset $S$ which contains the spacetranslation, space-rotation, and acceleration subgroups. By assumption, every member $A$ of $S$ is represented by an operator $U(A)$ which leaves the subalgebras of individual particles invariant. Does this imply that the representatives $U\left(T_{\tau}\right)$ of the time-translation subgroups with elements $T_{\tau}$ share the assumed property with the operators $U(A)$ ?

The answer is affirmative if the set $S$ generates the subgroup containing $\left\{T_{\tau}\right\}$, i.e. if for every element $T_{\tau}$ there exists an equation of the form

$$
\prod_{i} A_{i}=T_{\tau} . \quad\left(A_{i} \in S\right)
$$

Indeed, the representatives are then related by the equation

$$
\prod_{i} U\left(A_{i}\right)=U\left(T_{\tau}\right)
$$

and since every operator on the left-hand side leaves the sub-algebras invariant, so does $U\left(T_{\tau}\right)$.

Our assertion is that $S$ generates $\left\{T_{\tau}\right\}$ in the Poincaré group, but not in the Galilei group.

Proof: Consider the generators of the one-parameter subgroups in $S$. A linearly independent set consists of the three space-translation generators $(P)$, the three rotation generators $(J)$ and the three accelerations $(K)$. To obtain a closed Lie algebra which contains these generators we form the commutators of $P_{i}$ and $K_{j}$ and obtain the generators of time-translations [8]. Returning from the Lie algebra to the group, we see that every group which contains $S$ must also contain the time-translations $T_{\tau}$.

\section{References}

[1] Bakamjtan, B., and L. H. Thomas: Phys. Rev. 92, 1300 (1953).

[2] Foldy, L. L.: Phys. Rev. 122, 275 (1961). 
[3] Jordan, T. F., A. J. Macfarlane, and E. C. G. Sudarshan: Phys. Rev. 133, B. 487 (1964).

[4] Coester, F.: Helv. Phys. Acta 38, 7 (1965).

[5] Melvin, M. A.: Rev. Mod. Phys. 32, 477 (1960).

[6] Currie, D. G., T. F. Jordan, and E. C. G. Sudarshan: Rev. Mod. Phys. 35, 350 (1963).

[7] Davidon, W. C., and H. Ekstein: J. Math. Phys. 5, 1588 (1964).

[8] Hamermesh, M.: Group Theory and its applications to physical problems, p. 30. Reding, Mass.: Addison-Wesley 1962.

(Received November 1, 1964) 